\title{
OPTICAL AND MECHANICAL CHARACTERIZATION OF AN EVANESCENT COUPLER OPTICAL SWITCH
}

\author{
Marcel W. Pruessner ${ }^{1,2}$, Kuldeep Amarnath ${ }^{2}$, Madhumita Datta ${ }^{1,2}$, Daniel P. Kelly ${ }^{1,2}$, S. Kanakaraju ${ }^{3}$, \\ Ping-Tong $\mathrm{Ho}^{2}$, and Reza Ghodssi ${ }^{1,2}$ \\ ${ }^{1}$ MEMS Sensors and Actuators Laboratory (MSAL) and Institute for Systems Research (ISR), \\ ${ }^{2}$ Department of Electrical and Computer Engineering, University of Maryland, College Park, Maryland 20742 \\ ${ }^{3}$ Laboratory for Physical Sciences (LPS), College Park, Maryland 20740
}

\begin{abstract}
An InP-based optical switch with evanescent coupling mechanism is presented. Switching is achieved by electrostatic actuation of two parallel waveguides. Actuation at $5.4 \mathrm{~V}$ has been achieved with coupling efficiency of $60 \%$ resulting in $2.2 \mathrm{~dB}$ loss. The device exhibits $47 \mathrm{~dB}$ channel isolation in the off state and is compact with an active area of $500 \mu \mathrm{m} \times 5 \mu \mathrm{m}$.
\end{abstract}

\section{INTRODUCTION}

Indium phosphide (InP) is attractive for optical communications due to its direct bandgap and suitability as a substrate material for active optical devices operating at the $1550 \mathrm{~nm}$ wavelength. To date, InP-based MEMS efforts have mainly concentrated on tunable filters [1], likely due to the small displacements required for these types of devices. However, we previously demonstrated that InP is suitable for MEMS devices with several micron displacement range [2]. We also previously characterized suspended InP-based waveguides for use in optical MEMS [3] and now present an optical waveguide MEMS switch.

Contrary to free-space switches, which typically use relatively large mirrors, we are developing a moving waveguide switch. Compared to previously developed MEMS seriallycoupled waveguide switches $[4,5]$, our evanescent parallelcoupled switch is smaller and potentially enables variable power coupling so that it can also function as a variable power splitter/attenuator. Passive evanescent couplers are commonly used as optical power splitters. Active couplers have used thermal heating to perform optical switching by varying the refractive index, $n$, between two fixed parallel waveguides [6]. However, these devices typically draw significant current. Other approaches have utilized expensive electro-optic materials [7] to vary $n$ and achieve switching.

Our device uses electrostatic actuation to vary the spacing between two movable suspended waveguides, resulting in lowpower and relatively high-speed operation (compared to thermal couplers [6]). We chose InP due to its potential for optical gain at $1550 \mathrm{~nm}$. However, for passive operation our device is not limited to expensive semiconductors [7] and can be fabricated in relatively inexpensive materials - i.e. polymer waveguides on silicon actuators - without significant design changes.

\section{THEORY}

The MEMS coupler consists of two movable parallel waveguides that are electrostatically actuated until pull-in so as to vary the spacing between them (Figure 1). Optical switching then occurs by evanescent coupling and mode-interference. If two single-mode waveguides are brought close together, then the

Travel support has been generously provided by the Transducers Research Foundation and by the DARPA MEMS and DARPA BioFlips programs. compound waveguide structure can support two modes: even and odd [8]. These modes travel at different velocities (propagation constants, $\beta$ ) along the length $z$ of the waveguide. The optical power measured at the output will be the contribution of both even and odd modes, resulting in a constantly varying exchange of power between BAR and CROSS waveguides throughout the interaction length (Eq. 1). The coupling coefficient, $\kappa$, depends exponentially on the waveguide gap (Eq. 2), as well as on the waveguide geometry, refractive index and optical polarization.

$$
\begin{aligned}
& P_{\mathrm{BAR}}=P_{\mathrm{IN}} \cos ^{2}(\kappa \cdot z) \text { and } P_{\mathrm{CROSS}}=P_{\mathrm{IN}} \sin ^{2}(\kappa \cdot z) \\
& \kappa \propto \exp (-g a p)
\end{aligned}
$$

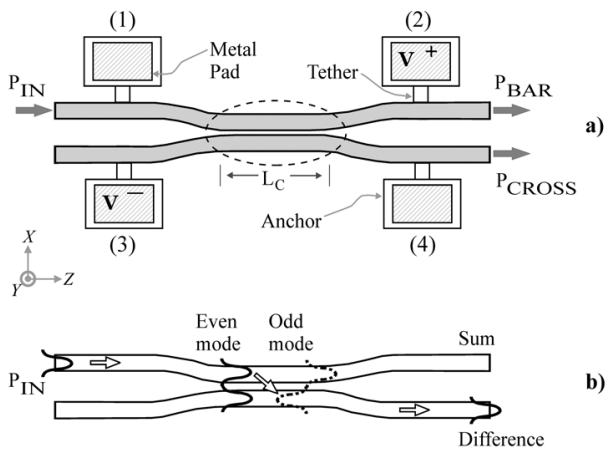

Figure 1. a) Top view schematic of coupler, b) evanescent coupling via mode interference. The $2 \mu \mathrm{m}$ wide waveguides are normally straight with 1 um gap before actuation.

\section{FABRICATION}

Processing of our device (Figure 2) is similar to that in Refs. $[2,3]$. We patterned $7000 \AA \mathrm{SiO}_{2}$ using positive resist, projection lithography, and reactive ion etching (RIE). The top $5 \mu \mathrm{m}(4 \mu \mathrm{m}$ waveguide layers and part of the $\operatorname{In}_{0.53} \mathrm{Ga}_{0.47} \mathrm{As}$ sacrificial layer) were etched by methane-hydrogen RIE, resulting in vertical sidewall profiles (88 degrees) and less than $50 \mathrm{~nm}$ roughness. After removal of the $\mathrm{SiO}_{2}$ mask, Ni-Ge-Au-Ni-Au ohmic contacts were deposited and patterned by lift-off followed by a 40 second rapid thermal anneal at $400{ }^{\circ} \mathrm{C}$. We cleaved the devices and etched the InGaAs layer in $\mathrm{HF}: \mathrm{H}_{2} \mathrm{O}_{2}: \mathrm{H}_{2} \mathrm{O}$ (1:1:8) with complete selectivity over InP followed by supercritical $\mathrm{CO}_{2}$ drying. A complete switch is shown in Figure 3.

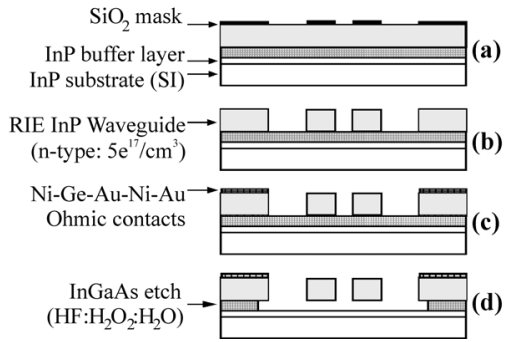

Figure 2. Process flow of the InP coupler optical switch. 


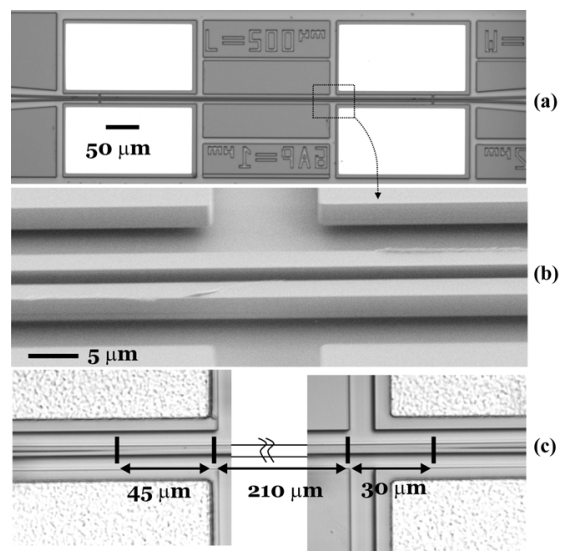

Figure 3. a) Fabricated coupler, b) close up of n-type suspended waveguides, c) measured coupling length: $L_{P U L L-I N}=285 \mu \mathrm{m}$.

\section{EXPERIMENTAL DETAILS}

Initial testing of the device was performed with S-polarized light (electric-field perpendicular to wafer plane) at $1550 \mathrm{~nm}$ wavelength. The experimental setup consisted of a tunable laser, a polarization control unit and input/output lensed fibers controlled by piezoelectric XYZ-stage. A probe station and function generator were used to actuate the device. Electrical actuation signals and optical output power were fed to an oscilloscope. We measured the BAR and CROSS outputs separately.

Prior to actuation, we measured less than $0.6 \mathrm{nW}$ of optical power in the CROSS waveguide for $30 \mu \mathrm{W}$ power in the BAR waveguide resulting in a channel isolation of $47 \mathrm{~dB}$ (in the 'off' state). Next, a $5.4 \mathrm{Vp}$-p, $10 \mathrm{~Hz}$ square wave with DC offset was used to modulate the waveguide gap and achieve pull-in during the optical measurements. The results in Figure 4 show $60 \%$ coupling of power from the BAR to the CROSS waveguide during pull-in (relative to $P_{B A R}$ in the uncoupled state), resulting in $2.2 \mathrm{~dB}$ coupling loss. The uncoupled BAR power at pull-in is $10 \%$.

Figure 5 shows the switching speed of the device in response to a $10 \mathrm{~Hz}$ square wave actuation pulse. The rise- and falltime is $1 \mathrm{~ms}$. However, there is a $400 \mu$ s delay in the risetime. This delay is due to the exponential dependence of evanescent coupling on the waveguide separation (Eq. 2): the $400 \mu$ s delay is the time required for the beams to come into close proximity in order for coupling to occur. The beams separate immediately after removal of the actuation voltage so that the falltime does not exhibit this delay in the response. It turns out that the switching speed is dominated by thermal actuation, as discussed below.

(a)

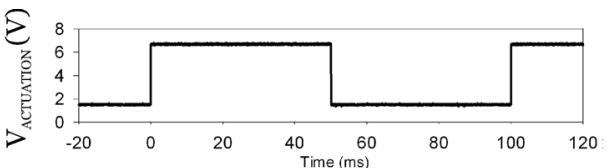

(b)

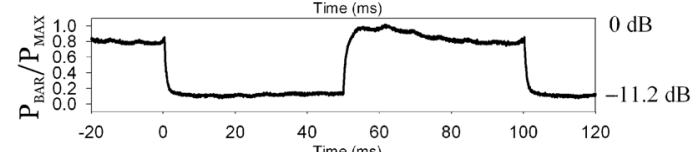

(c)

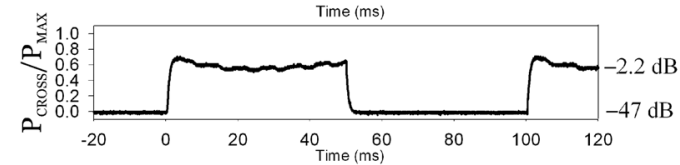

Figure 4. a) Actuation signal, b) normalized BAR, and c) normalized CROSS signals, where $P_{M A X}=$ maximum $B A R$ power (S-polarization: electric field $E=E_{Y}, \lambda=1550 \mathrm{~nm}$ wavelength). (a)

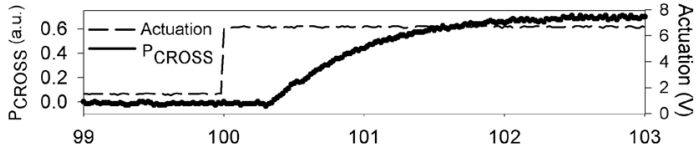

(b)

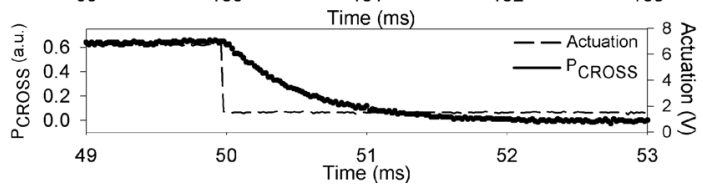

Figure 5. Measured a) risetime and b) falltime. The speed is $t_{\text {SWITCH }}<1.4 \mathrm{~ms}\left(S\right.$-polarization: $\left.E=E_{Y}, \lambda=1550 \mathrm{~nm}\right)$.

\section{THERMAL ISSUES}

The $1.4 \mathrm{~ms}$ total switching time (Figure 5) is slow compared to typical electrostatic pull-in actuators: the reason is that the tested device was actually thermally actuated. Figure 2 a shows a $0.45 \mu \mathrm{m}$ thick InP buffer layer grown on top of a semi-insulating (SI) InP substrate. The buffer layer is typically grown before any additional epilayers in order to obtain uniform surface conditions for crystal growth. Undoped InP as grown by MBE is n-type conducting with $\mathrm{n} \sim 10^{16} / \mathrm{cm}^{3}$. This results in significant substrate leakage currents. In Figure 1a a voltage is applied between terminals 2 and 3 to actuate the device. The measured resistance between terminals 1 and 3 was $R_{13}=R_{\text {LEAKAGE }} \cong 300 \Omega$, and the waveguide resistance (i.e. terminals 1 and 2) was around $5 \mathrm{k} \Omega$ (measured on separate samples without the InP buffer layer, but with similar waveguides and doping). Therefore, terminals 1 and 3 are at similar potential and significant current flows through the waveguide resulting in a combination of electrostatic and thermal actuation. Measurements have shown that $2.5 \mathrm{~mW}$ of thermal power are dissipated in the waveguides during actuation at $5.4 \mathrm{~V}$.

Simple calculations shed some insight on the electro-thermal behavior of our device. The induced (thermal) strain required to actuate the waveguides is [2]:

$$
\varepsilon_{\text {THERMAL }}=-\left[\frac{\pi^{2}}{4}\left(\frac{d x}{L}\right)^{2}+\frac{\pi^{2}}{3}\left(\frac{w}{L}\right)^{2}+\varepsilon_{O}\right]
$$

where $d x=0.5 \mu \mathrm{m}$ is the actuation distance of each waveguide during switching, $L=500 \mu \mathrm{m}$ is the waveguide length, and $w=2 \mu \mathrm{m}$ is the waveguide width. The first term represents the induced axial strain, the second is the Euler buckling strain, and $\varepsilon_{0}$ is the intrinsic tensile strain of the waveguides. Assuming $\varepsilon_{\mathrm{o}}=0.04 \%$ (the designed intrinsic tensile strain), the necessary thermal strain is $\varepsilon_{\text {THERMAL }}=-0.0455 \%$. The coefficient of thermal expansion is $\alpha=4.56 \times 10^{-6} / \mathrm{K}$ [9], which implies a temperature increase of $\Delta T=\varepsilon_{\text {THERMAL }} / \alpha=100 \mathrm{~K}$ in order to thermally actuate the switch. Based on our calculations and the measured switching speed and heat dissipation, we conclude that thermal heating is the dominant actuation mechanism, not pull-in.

The thermal effects on the characteristics of our device are discussed below. Future devices will use epiwafers without an InP buffer layer and with pn-junctions between waveguide and sacrificial layers to avoid substrate leakage currents.

\section{OPTICAL CHARACTERIZATION}

After the initial experiments, we repeated our measurements with P-polarized light (E-field parallel to wafer plane), again at $1550 \mathrm{~nm}$ wavelength. In these experiments we obtain $10 \%$ coupling, significantly less than for S-polarization (Figure 6). 
(a)

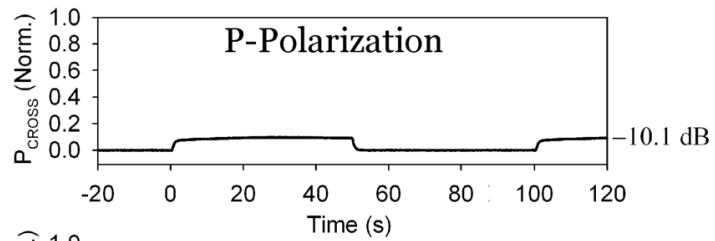

(b)

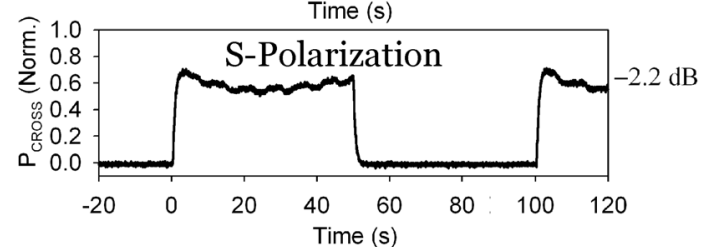

Figure 6. Experimentally measured coupling efficiency to CROSS waveguide: a) P-polarization: $10 \%$, and b) S-polarization: $60 \%$.

For our optical analysis we approximate the coupling length ( $L_{\mathrm{C}}$ in Figure 1) as the pull-in length, which equals $285 \mu \mathrm{m}$ (Figure 3c). If we know the coupling coefficient $\kappa$, then we can obtain the coupled power $\left(P_{\mathrm{CROSS}}\right)$ using Eq. 1 . We can obtain the coupling coefficient $\kappa$ by simulation. While it is difficult to precisely know the waveguide gap during actuation, we assume the switching gap to be greater than twice our maximum sidewall roughness $(>2 \times 50 \mathrm{~nm}=100 \mathrm{~nm})$. This gives us an estimate and starting point for the gap in our simulations.

Polarization-dependent coupling was analyzed by simulating [10] the effective refractive index $\left(\mathrm{n}_{\text {effective }}\right)$ for even and odd modes for both S- and P-polarizations for various gaps (Figure 7). From $n_{\text {effective }}$ we calculate the coupling coefficient [8]:

$$
\kappa=\left(\beta_{\text {even }}-\beta_{\text {odd }}\right) / 2=\left(n_{\text {even }}-n_{\text {odd }}\right) k_{o} / 2,
$$

where $k_{o}=2 \pi / \lambda_{\mathrm{o}}$ and $\lambda_{\mathrm{o}}=1550 \mathrm{~nm}$. After simulations for various gaps, we find that a $120 \mathrm{~nm}$ separation between the waveguides at pull-in gives us the best agreement between simulation and experiment (Table 1). Using a $120 \mathrm{~nm}$ gap and a coupling length of $L=285 \mu \mathrm{m}$ (as measured in Figure 3c) we expect $54.7 \%$ and $1.07 \%$ coupling for the S- and P-polarizations, respectively (Figure 8), similar to the $60 \%$ obtained experimentally for Spolarization, but significantly smaller than our measured $10 \%$ for P-polarization (Figure 6).

A possible source of discrepancy between our measurements and simulation is that the exact waveguide gap at pull-in is not known. Also, our waveguides are not strictly single-mode. The $2 \mu \mathrm{m} \times 2 \mu \mathrm{m}$ waveguide core cross-section (Figure 7) was chosen for mechanical robustness. Subsequent simulations have shown that the core cross-section should be less than $0.7 \mu \mathrm{m} \times 0.7 \mu \mathrm{m}$ for single-mode behavior. This explains the larger than expected measured coupled power for P-polarization due to higher order mode coupling.

Another concern is that thermal actuation of our waveguides will cause a shift in the refractive index. The temperature dependence of the refractive index for $\operatorname{InP}$ is $\mathrm{d} n / \mathrm{d} T=1.9 \times 10^{-4} / \mathrm{K}$ at $1550 \mathrm{~nm}$ wavelength [9], resulting in an increase of $1.9 \%$ in the refractive index during thermal actuation (assuming $\Delta T=100 \mathrm{~K}$ ). Repeating our simulations $[\mathbf{1 0}]$ for a $2 \%$ increase in the refractive index, we obtain $P_{\text {CROSS }}=49.0 \%$ for S-polarized light and $P_{\text {CROSS }}=0.86 \%$ for P-polarized light, similar to the results in Table 1. We conclude that the thermally-induced increase in the refractive index of our waveguides does not significantly change our coupling analysis.

While our analysis assumes single-mode waveguides (the waveguides support more than one mode), the general agreement between measured and simulated coupling efficiency for different polarization is insightful for future device design and suggests that our device behavior is dominated by the fundamental mode. Future devices, however, should utilize single-mode waveguides to ensure fundamental mode-coupling.

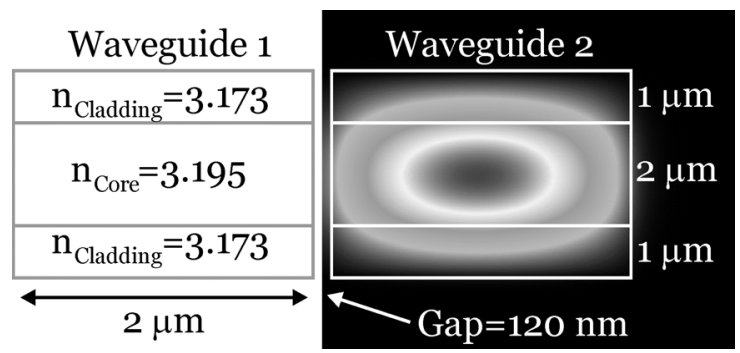

Figure 7. Half-structure cross-section simulation (right) of the optical mode in the coupler from which $n_{\text {effective }}$ is obtained [10].

\begin{tabular}{|c|c|c|}
\hline Gap=120 nm & P-Polarization & S-Polarization \\
\hline $\mathbf{n}_{\text {effective }}$ (even) & 3.163234 & 3.166794 \\
\hline $\mathbf{n}_{\text {effective }}(\mathbf{o d d})$ & 3.163054 & 3.165353 \\
\hline $\boldsymbol{\kappa}\left(\boldsymbol{\mu \mathbf { m } ^ { - 1 } )}\right.$ & $3.648300 \times 10^{-4}$ & $2.920668 \times 10^{-3}$ \\
\hline $\mathbf{P}_{\mathbf{C R O S S}, \mathbf{L}=\mathbf{2 8 5} \boldsymbol{\mu m}} \mathbf{( \% )}$ & 54.7 & 1.07 \\
\hline
\end{tabular}

Table 1. Simulated Coupling for S- and P-Polarizations.

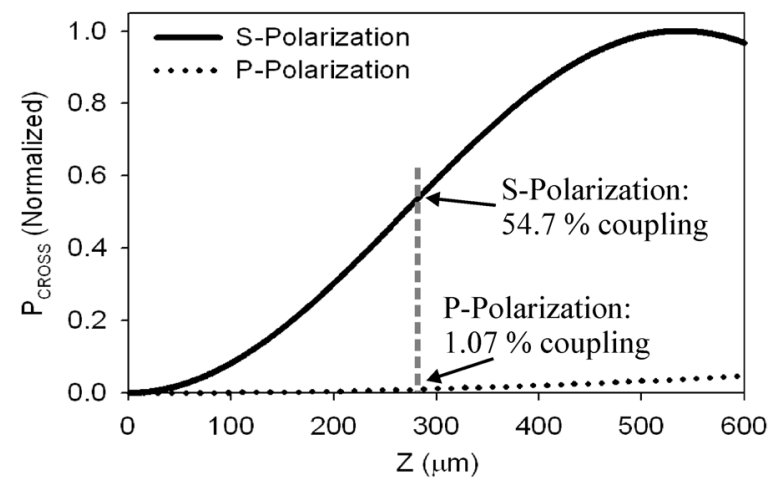

Figure 8. Predicted coupling for S- and P-polarizations vs. coupling length, neglecting losses. For $285 \mu \mathrm{m}$ coupling length (dashed vertical line), P-polarization yields $1.07 \%$ and $S$ polarization gives $54.7 \%$ coupling.

\section{MECHANICAL CHARACTERIZATION}

The complete coupler switch does not simply consist of the device shown in Figures 1 and 3. Rather, it has sets of optical inputs and outputs connected to the coupled-region via S-curved waveguides (Figure 9). These S-curved waveguides are suspended in air by $1.5 \mu \mathrm{m}$ wide, $5 \mu \mathrm{m}$ long tethers using the same 'suspended waveguide' technology presented in [3]. The effects of tether compliance were studied by experiment and simulation.

We gauged tether compliance by measuring the displacement of the S-curve region during actuation of the two waveguides in the coupled region (Figure 9). An ideal tether would be completely rigid and would result in zero movement of the Scurve. Measurements were performed using an optical profilometer [11] while actuating the coupled waveguides. We measured $700 \mathrm{~nm}$ total S-curve displacement for $V_{\text {ACTUATION }}=5 \mathrm{Vp}$-p at $800 \mathrm{~Hz}$ (Figure 10$)$. Note that this did not result in pull-in of the coupled-waveguide region. 


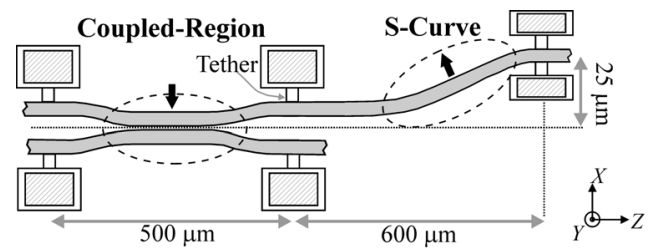

Figure 9. Actuation of the coupled region causes oscillation of the S-curve due to compliance and the pivot action of the tether.

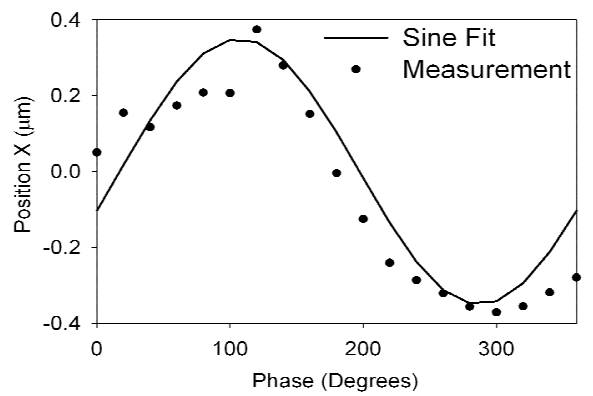

Figure 10. Measured x-direction displacement of the S-curve (due to thermal effects) in response to a sine wave actuation signal $(5 \mathrm{Vp}-\mathrm{p}, 800 \mathrm{~Hz})$. Electrostatic actuation should limit this effect.

Static load ANSYS simulations showed that while we expect only small tether movement $(<3 \mathrm{~nm})$ during actuation, the tethers also rotate slightly, resulting in significant displacement $(\sim 150 \mathrm{~nm})$ of our S-curves. However, this is still much less than the $700 \mathrm{~nm}$ S-curve displacement measured experimentally. The additional movement of the S-curve is the result of thermal expansion of the coupled waveguides during actuation.

Future devices will rely on electrostatic rather than thermal actuation by preventing substrate leakage currents. Based on ANSYS simulations, this should prevent any significant movement of the S-curve waveguides and will result in more efficient actuation in terms of both power consumption and switching speed. Initial pull-in actuation of devices fabricated on new epiwafers (without InP buffer layer and including pn-junctions between waveguide and sacrificial layers) result in switching times as low as $30 \mu \mathrm{s}$ based on electrical measurements and pull-in voltages as low as $3.7 \mathrm{~V}$. This performance is comparable to that of serially-coupled MEMS waveguide switches $[4,5]$, but with much smaller device area. The substrate leakage is negligible with measured $R_{13}$ and $R_{24}$ (Figure 1 ) in the tens of $\mathrm{M} \Omega$ range. This reduces the power consumption to the $\mu \mathrm{W}$-range, significantly smaller than for thermal couplers [6]. A concern with pull-in actuators, however, is that they are prone to stiction, posing a significant concern that needs to be addressed [12].

\section{CONCLUSIONS}

We have presented an InP-based optical MEMS switch with evanescent coupling mechanism in which we control the spacing between two semiconductor waveguides by application of a voltage. The device is compact, low voltage, relatively low loss and enables dense integration on a chip. We believe it can find applications in photonic circuits and devices, including tunable filters [13]. Other applications include optical network restoration in which switching speeds of $1 \mathrm{~ms}$ are more than adequate.

Although designed for electrostatic pull-in actuation, the current device exhibits thermal actuation due to substrate leakage currents. Future devices will be based on electrostatic pull-in, resulting in more efficient switches with lower power consumption and expected switching speeds in the sub-100 $\mu$ s range. While the present waveguides are multi-mode, the demonstrated device can be scaled down in dimensions (i.e. reducing the waveguide crosssection) to obtain single-mode waveguides without significant change in the design. We expect that reducing the device size will further decrease the switching time. Stiction and reliability is a concern, however. Future devices could utilize actuators that do not exhibit pull-in, thereby increasing the device reliability. In this manner, variable coupling can also be achieved so that the device acts as a switch and as a variable power splitter or attenuator.

\section{ACKNOWLEDGMENTS}

The authors especially thank Mr. L. C. Calhoun for assistance with the MBE wafer growth, and Mr. L. C. Olver and the Laboratory for Physical Sciences (LPS) staff for access to the cleanroom facilities. This work is supported by a National Science Foundation (NSF) CAREER award (R. Ghodssi) and by the LPS.

\section{REFERENCES}

1. S. Irmer, J. Daleiden, V. Rangelov, C. Prott, F. Römer, M. Strassner, A. Tarraf, and H. Hillmer, "Ultralow Biased Widely Continuously Tunable Fabry-Pérot Filter," IEEE Phot. Tech. Lett. 15 (3) 434-436 (2003).

2. M. W. Pruessner, T. T. King, D. P. Kelly, L. C. Calhoun, and R. Ghodssi, "Mechanical property measurement of InP for optical communications," Sens. Actuators A, 105 (2), 190-200, (2003).

3. D. Kelly, M. W. Pruessner, K. Amarnath, M. Datta, S. Kanakaraju, L. C. Calhoun, and R. Ghodssi, "Monolithic Suspended Optical Waveguides for InP MEMS," IEEE Phot. Tech. Lett., in press, May 2004.

4. E. Ollier, "Optical MEMS devices based on moving waveguides,” IEEE J. Sel. Top. Quan. Elec., 8, 155-162, (2002).

5. T. Bakke, C. P. Tigges, J. J. Lean, C. T. Sullivan, and O. B. Spahn, "Planar microoptomechanical waveguide switches," IEEE J. Sel. Top. In Quan. Elec., 8 (1), 64-72 (2002).

6. Q. Lai, W. Hunziker, and H. Melchior, "Low-Power Compact $2 \times 2$ Thermooptic Silica-on-Silicon Waveguide Switch with Fast Response," IEEE Phot. Tech. Lett., 10 (5), 681-683 (1998).

7. V. R. Chinni, T. C. Huang, P. K. A. Wai, C. R. Menyuk, and G. J. Simonis "Performance of Field-Induced Coupler Switches," IEEE J. Quant. Elec., 31 (11), pp. 2068-2074 (1995).

8. Katsunari Okamoto, "Fundamentals of Optical Waveguides," Academic Press: San Diego (2000).

9. E. Gini and H. Melchior, "Thermal Dependence of the Refractive Index of InP Measured with Integrated Optical Demultiplexer," J. Appl. Phys., 79 (8), pp. 4335-4337 (1996).

10. Apollo Photonics, OWMS 1.21: Optical Waveguide Mode Solver, Waterloo, Canada.

11. Model: Wyko NT1100 with dynamic MEMS (DMEMS) option, available from Veeco Instruments, Woodbury, New York.

12. H. Lim, C. Carraro, R. Maboudian, M. W. Pruessner, and R. Ghodssi, "Chemical and Thermal Stability of Alkanethiol and Sulfur Passivated InP(100)," Langmuir, 20, pp. 743-747, 2004.

13. M.-C. M. Lee and M. C. Wu, "A MEMS actuated tunable microdisk resonator," Proc. 2003 IEEE/LEOS Conf. on Optical MEMS, Hawaii, (2003), pp. 28-29. 